\title{
The Inhibitory Effects of Anthocyanin-Rich Sunrouge Tea on Pancreatic Lipase Activity
}

\author{
Nobuya Shirai*
}

National Agriculture and Food Research Organization, Institute of Fruit Tree and Tea Science, 2769 Kanaya, Shimada, Shizuoka 428-8501, JAPAN

\begin{abstract}
This study investigated the in vitro and in vivo effects of Sunrouge (SR), which is an anthocyaninrich green tea. Hot and cold water extracts (CWEX) of Yabukita (YK; regular green tea), SR second crop (SR2), and SR third crop (SR3) were prepared. The 50\% inhibitory concentrations for YK, SR2, and SR3 CWEXs against pancreatic lipase in vitro were $12.3 \pm 2.8,6.2 \pm 0.3$, and $4.0 \pm 1.1 \mathrm{mg} / \mathrm{mL}$, respectively. Epigallocatechin gallate (EGCG) accounted for $4.3 \%, 6.0 \%$, and $6.3 \%$ of YK, SR2, and SR3 CWEXs, respectively. SR2 had the highest anthocyanin content of these three samples. In vivo, the increase in the plasma triacylglycerol (TG) concentration following oral administration of oil to mice was significantly suppressed at 60 and $120 \mathrm{~min}$ in animals treated with SR2. No significant differences were observed between the plasma TG concentration in the YK and control groups. These results suggested that concomitant administration of SR with oil may suppress lipid absorption and that EGCG may exert this effect.
\end{abstract}

Key words: lipid, green tea, obesity, functional food, epigallocatechin gallate

\section{INTRODUCTION}

Obesity presents an important challenge to human health because it increases the risk of diabetes and hypertension ${ }^{1,2)}$. Many studies have shown that some foods and food components can contribute to the prevention of obesity $^{1,3-6)}$. The consumption of green tea may contribute to the prevention of obesity by increasing fat oxidation and inhibiting fat absorption ${ }^{7)}$. Recently, a new variety of green tea was developed by crossing Camellia taliensis with Camellia sinensis and registered as Sunrouge(SR). SR tea has higher levels of anthocyanins than other more common green tea varieties such as Yabukita(YK); these are effective for the prevention of eyestrain ${ }^{8)}$ and have antioxidant activity $^{9-11)}$. Furthermore, SR also contains similar levels of catechins such as epigallocatechin gallate(EGCG) as other green tea varieties ${ }^{12}$. On the other hand, green tea leaves including SR have the other useful components such as fat-soluble vitamins. These should also promote health and some food products using this novel functional food have been marketed recently. The present study investigated the effects of SR on lipase activity and fat absorption for the obese prevention and promotion of the health.

\section{EXPERIMENTAL}

\subsection{Samples}

Dried YK and SR puree powders were supplied by Nepure Corporation(Tokyo, Japan). SR2 was produced from the second picking of the tea crop grown at Tokunoshima in 2012. SR3 was made from the third picking of the tea crop grown at Makurazaki in 2012.

\subsection{Chemicals}

Chloroform, ethyl acetate, safflower oil, methanol, 2,2,4-trimethypentan, phosphoric acid, copper sulfate, cholic acid, triolein, and phosphatidylcholine were purchased from Wako Pure Chemical Industries, Ltd. (Osaka, Japan). Lipase was purchased from Tokyo Chemical Industry Co. Ltd. (Tokyo, Japan).

2.3 Preparation and fractionation of SR water extract (WEX)

A cold water extract (CWEX) of each dried puree powder was prepared by adding 20 volumes of water, incubating at $6^{\circ} \mathrm{C}$ for $3 \mathrm{~h}$, centrifuging at $4^{\circ} \mathrm{C}(3000 \mathrm{~g}, 10 \mathrm{~min})$, and then collecting the supernatant. A hot water extract(HWEX) of each dried puree powder was prepared by adding 20 volumes of boiled distilled water, incubating for $3 \mathrm{~min}$, and collecting the liquid using vacuum filtration. The CWEX

\footnotetext{
*Correspondence to: Nobuya Shirai, National Agriculture and Food Research Organization, Institute of Fruit Tree and Tea Science, 2769 Kanaya, Shimada, Shizuoka 428-8501, JAPAN

E-mail: nshinya@ affrc.go.jp

Accepted July 31, 2017 (received for review June 20, 2017)

Journal of Oleo Science ISSN 1345-8957 print / ISSN 1347-3352 online

http://www.jstage.jst.go.jp/browse/jos/ http://mc.manusriptcentral.com/jjocs
} 
and HWEX were then lyophilized.

An ethyl acetate extraction fraction (EEX) was also prepared from the CWEX. The CWEX was dissolved by 20 volumes distilled water and added the same volume of chloroform, shaking vigorously, centrifuging at $650 \mathrm{~g}$ for 10 min, and removing the lower layer. This chloroform extraction was repeated twice. The residue of the CWEX solution was then shaken vigorously with an equal volume of ethyl acetate, which was recovered after centrifugation at $650 \mathrm{~g}$ for $10 \mathrm{~min}$. This extraction was repeated 4 times prior to evaporation and lyophilization of the EEX.

\subsection{Analysis of polyphenols}

The total anthocyanin concentration was measured using the $\mathrm{pH}$ differential method ${ }^{13)}$. The catechins were separated on a reverse phase C18 column $(4.0 \mathrm{~mm} \times 150 \mathrm{~mm}, 5 \mu \mathrm{m}$ Wakopack Navi 18C5; Wako Pure Chemical Co. Ltd.) at $35^{\circ} \mathrm{C}$, and detected by an ultraviolet absorption detector (UV-880; JASCO Co. Ltd., Tokyo, Japan) at $280 \mathrm{~nm}$. The flow rate was $1.2 \mathrm{~mL} / \mathrm{min}$ and an elution gradient was employed using $0.05 \%$ phosphoric acid and methanol; the mobile phase composition of methanol changed linearly over time, with $10 \%$ at $0-5 \mathrm{~min}, 10-32 \%$ between 5 and 35 min, and $32-40 \%$ between 35 and 40 min.

\subsection{Lipase inhibitory activity}

Lipase inhibition was determined using a previously described method, with some modification ${ }^{14)}$. The substrate solution comprised triolein (80 mg), phosphatidylcholine (10 mg), and cholic acid (5 mg) in $9 \mathrm{~mL} 0.1 \mathrm{M}$ Tris buffer $(\mathrm{pH}, 8.0)$; this was mixed by vortexing. For the assay of lipase inhibitory activity in the presence of casein, the 0.1 M Tris buffer $(\mathrm{pH}, 8.0)$ was replaced by $0.5 \%(w / v)$ casein in $0.1 \mathrm{M}$ Tris buffer $(\mathrm{pH}, 8.0)$. Casein was dissolved in this buffer at $150^{\circ} \mathrm{C}$. The substrate solution $(0.1 \mathrm{~mL})$ and sample solution $(0.1 \mathrm{~mL})$ were mixed and preincubated for $5 \mathrm{~min}$ at $37^{\circ} \mathrm{C}$ before adding $0.05 \mathrm{~mL}$ pancreatic lipase solution (50 $\mathrm{mg} / \mathrm{mL}$ ). Following incubation at $37^{\circ} \mathrm{C}$ for $30 \mathrm{~min}$, the reactions were stopped by the addition of $3 \mathrm{~mL}$ mixed solvent (chloroform : 2,2,4-trimethylpentane : methanol; 50:49.9:0.1). All tubes were shaken vigorously prior to adding the copper reagent $(1 \mathrm{~mL})$, shaking again, and centrifuging at $1000 \mathrm{~g}$ for $10 \mathrm{~min}$. Part of the upper organic phase $(0.5 \mathrm{~mL})$ was collected and reacted with $0.5 \mathrm{~mL}$ bathocuproine in chloroform $(1 \mathrm{~g} / \mathrm{L})$ containing $0.5 \mathrm{~g} / \mathrm{L}$ hydroxyanisole. The absorbance was measured at $480 \mathrm{~nm}$. Lipase inhibitory activity was calculated using the following formula:

Lipase inhibitory activity $(\%)=($ [lipase blank $]-$ [Sample $]) /([$ lipase blank $]-[$ Blank $]) \times 100$

[lipase blank] : absorbance after the incubation with lipase and without sample at $37^{\circ} \mathrm{C}$ for $30 \mathrm{~min}$.

[Sample]: absorbance after the incubation with lipase and sample at $37^{\circ} \mathrm{C}$ for $30 \mathrm{~min}$
[Blank]: absorbance after the incubation without lipase and sample at $37^{\circ} \mathrm{C}$ for $30 \mathrm{~min}$

The $50 \%$ inhibitory concentrations were calculated using linear regression $(n=3)$.

\subsection{Animal Study}

Male Crlj : CD-1 (ICR) strain mice (5 weeks old) were obtained from Charles River Japan Inc. (Atsugi, Kanagawa, Japan). The animals received MF diet (Certified Diet: Oriental Yeast Co., ltd., Tokyo, Japan) and were maintained at $24.5^{\circ} \mathrm{C}$ with a relative humidity of $60 \%$. Room lighting consisted of 12-h periods of light and dark. The mice received diet and water ad libitum until $12 \mathrm{~h}$ prior to treatment administration, when they were fasted. The animals were orally administered $1 \mathrm{~mL}$ sample solution/kg body weight. The treatments comprised $16.7 \%(v / v)$ safflower oil and $8 \%$ dried YK or SR2 puree powder in citric acid buffer $(\mathrm{pH}$, 5.2 ), or vehicle. The test sample were not emulsified, but were furiously mixed just before the dosage. SR2 is anthocyanin-rich sample and strongly shows a characteristic of SR. Therefore, I have used the SR2 in this animal study. Animals were dissected under isoflurane anesthesia at 0 , 30, 60, or 120 min after dosing. Oil and YK + oil groups used four mice and SR + oil group used five mice in each time. Initial time used three mice. Blood was collected from the inferior vena cava using a heparinized syringe, and placed in ice-cold tubes. The plasma was separated by centrifugation at $3500 \mathrm{~g}$ for $2 \mathrm{~min}$ at $4^{\circ} \mathrm{C}$. The plasma triacylglycerol(TG), non-esterified fatty acid (NEFA), and glucose levels were measured by Wako commercial kits. This animal experiment was conducted in accordance with the guidelines for experimental animals of the National Agriculture and Food Research Organization, Japan.

\subsection{Statistical analysis}

The results were expressed as the mean \pm standard deviation. Differences between the control and experimental groups were assessed by Dunnett' s test using the EkuseruToukei 2010 software (Social Survey Research Information Co. Ltd.) .

\section{RESULTS}

\subsection{Recovery ratio of each fraction}

The recovery ratios of CWEX from dried YK, SR2, and SR3 puree powders were $23.9 \pm 0.5 \%, 23.7 \pm 0.6 \%$, and $24.3 \pm 0.7 \%$, respectively. The recovery ratios of EEX from lyophilized CWEX of YK, SR2, and SR3 puree were $20.0 \pm$ $1.8 \%, 21.5 \pm 1.4 \%$, and $18.9 \pm 1.6 \%$, respectively. The recovery ratios of HWEX from YK, SR2, and SR3 puree were $26.2 \pm 1.8 \%, 26.6 \pm 2.2 \%$, and $28.6 \pm 1.2 \%$, respectively. 


\subsection{Catechin and anthocyanin concentrations}

The concentrations of the major catechins and anthocyanin in CWEX, HWEX, and EEX of YK, SR2, and SR3 are shown in Table 1. The EGCG and epicatechin gallate (ECG) concentrations were lower in all three extracts of YK, as compared with those of SR2 and SR3. In contrast, the CWEX, HWEX, and EEX of SR2 and SR3 contained less epigallocatechin and epicatechin than the equivalent YK extracts. Anthocyanin was only detected in the SR extracts and its concentration was higher in SR2 than in SR3.

\subsection{The lipase inhibitory activity of each extract}

The 50\% inhibitory concentrations of the CWEXs of YK, SR2, and SR3 were 12.3 $\pm 2.8,6.2 \pm 0.3$, and $4.0 \pm 1.1 \mathrm{mg} /$ $\mathrm{mL}$, respectively. For the corresponding HWEXs, these values were $7.8 \pm 0.3,4.2 \pm 0.0$, and $3.4 \pm 0.4 \mathrm{mg} / \mathrm{mL}$, respectively. The ratios of the lipase inhibition produced by the same concentration of each EEX or CWEX in the presence or absence of casein are shown in Fig. 1. In the absence of casein, the CWEXs of SR2 and SR3 produced significantly greater lipase inhibition than the CWEX of YK; however, only SR3 showed significantly greater lipase inhibitory activity in the presence of casein. The EEXs of SR2 and SR3 tended to inhibit lipase activity more effectively than that of YK.

\subsection{Plasma lipid concentrations in mice after oral admin- istration}

The plasma TG and NEFA concentrations of mice following oral administration of vehicle, YK, or SR2 are displayed in Fig. 2. The increment in the plasma TG concentration after oral dosing was significantly suppressed in the SR2 group at 60 and $120 \mathrm{~min}$, as compared with the control group. The plasma NEFA concentration in the SR2 group was significantly lower than that of the control group at 120 min. No significant differences were observed between these plasma parameters in the $\mathrm{YK}$ and control groups.
Table 1 The catechin and anthocyanin concentrations within the indicated extracts of YK, SR2, and SR3 (w\%).

\begin{tabular}{lccc}
\hline & YK & SR2 & SR3 \\
\hline CWEX & & & \\
EGC & 10.0 & 5.6 & 4.7 \\
EGCG & 4.3 & 6.0 & 6.3 \\
EC & 2.4 & 2.1 & 1.1 \\
ECG & 1.1 & 1.9 & 1.4 \\
Anthocyanin & - & 0.05 & 0.03 \\
EEX & & & \\
EGC & 29.0 & 15.6 & 15.9 \\
EGCG & 17.1 & 24.1 & 27.7 \\
EC & 9.9 & 7.6 & 6.3 \\
ECG & 3.9 & 7.0 & 6.1 \\
Anthocyanin & - & 0.07 & 0.05 \\
HWEX & & & \\
EGC & 13.4 & 5.6 & 5.2 \\
EGCG & 10.0 & 10.3 & 12.1 \\
EC & 3.0 & 2.6 & 1.6 \\
ECG & 2.1 & 3.1 & 2.6 \\
Anthocyanin & - & 0.19 & 0.13 \\
\hline CWEX: cold & & & \\
\hline
\end{tabular}

CWEX: cold water extracts

EEX: ethyl acetate extraction fraction

HWEX: hot water extracts

EGC: epigallocatechin

EGCG: epigallocatechin gallate

EC: epicatechin

ECG: epicatechin gallate

- : trace or not detected

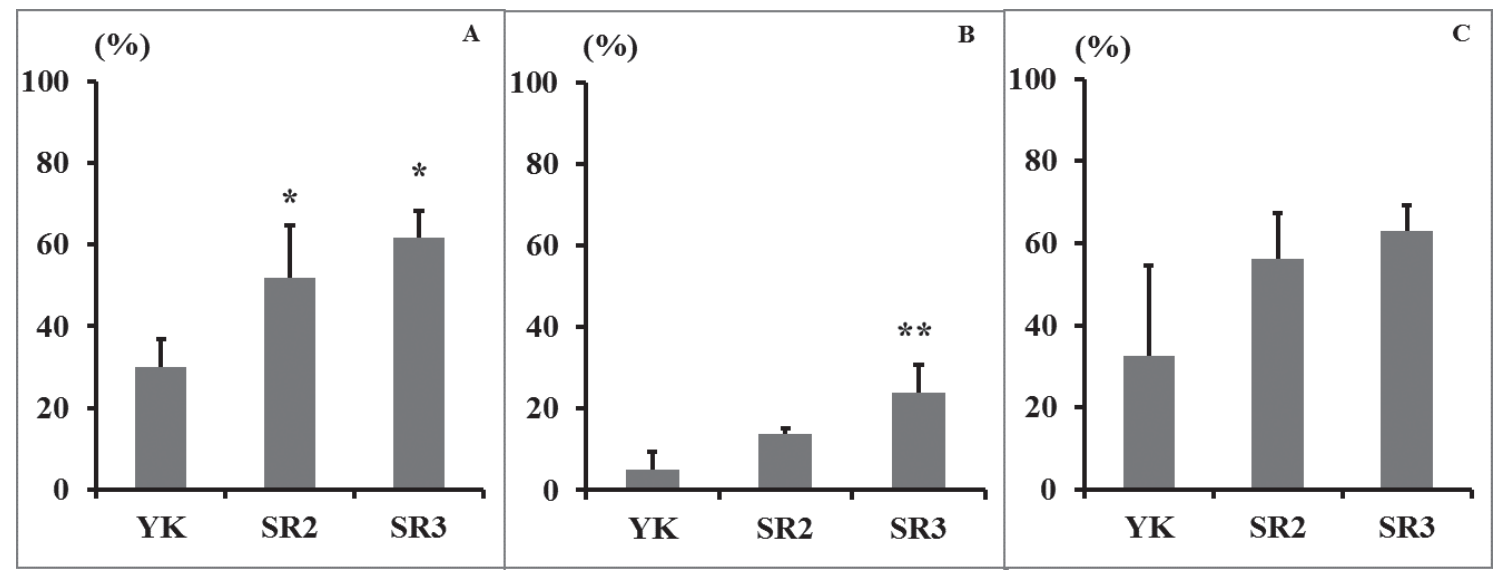

Fig. 1 The extent of lipase inhibition by CWEXs and EEXs of YK, SR2, and SR3. A: CWEX; B: CWEX + casein; C: EEX. The same concentration $(6 \mathrm{mg} / \mathrm{mL})$ of each extract was employed. ${ }^{*} p<0.05$, ${ }^{*} p<0.01$ for the comparison with YK. 


\section{N. Shirai}
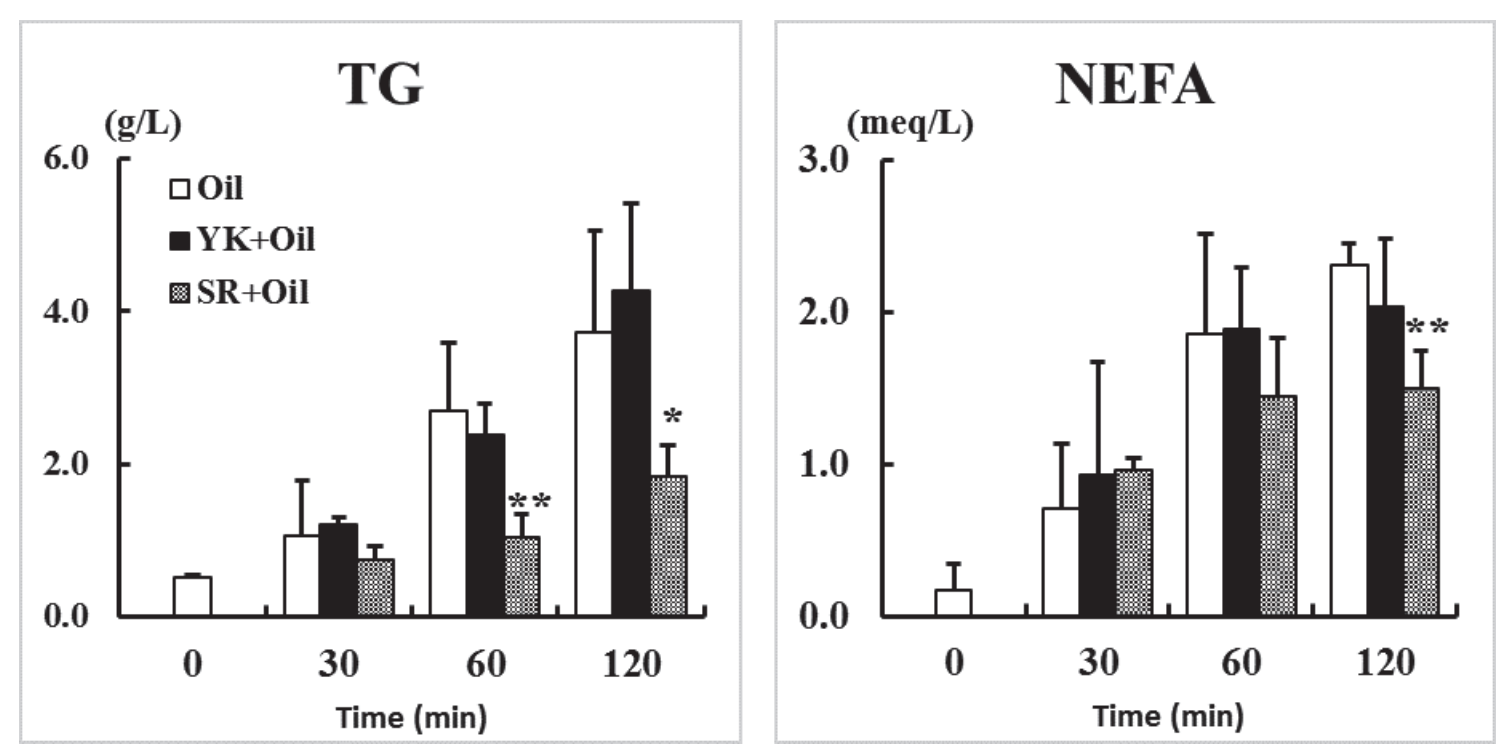

Fig. 2 The plasma TG and NEFA concentrations in mice after oral administration of oil, with and without YK or SR2. ${ }^{*} p<0.05,{ }^{*} p<0.01$ for the comparison with mice treated with oil only.

\section{DISCUSSION}

In this study, CWEXs and HWEXs of SR2 and SR3 showed greater lipase inhibitory activities than those of YK. EGCG and ECG have previously been shown to produce strong inhibitory effects on lipase activity ${ }^{1,15)}$. CWEXs of SR2 and SR3 contained higher levels of EGCG and ECG than did the CWEX of YK. Furthermore, the lipase inhibitory activity of each CWEX was down-regulated in the presence of casein. Gallate-type catechins such as EGCG bind to proteins ${ }^{16)}$. Wu et al. reported that EGCG inhibited lipase activity by forming a noncovalent bond with the lipase protein ${ }^{17)}$. These findings implied that the inhibitory activity of CWEXs of SR2 and SR3 may be associated with these gallate-type catechins. However, even though the percentages of EGCG and ECG in HWEXs of SR and YK did not differ significantly, SR2 and SR3 produced greater inhibitory effects than YK. These results suggested that SR may include other components with lipase inhibitory activities. A previous study indicated that Camellia taliensis contained 1,2-diO-galloyl-4,6-O- (S) -hexahydroxydiphenoyl- $\beta$-D-glucose ${ }^{18)}$. Similar hexahydroxydiphenoyl compounds have shown lipase inhibitory activities ${ }^{19)}$. A previous report showed that SR also contained a small amount of EGCG-3-O-methyl ${ }^{12)}$. Benifuuki green tea, which contains high levels of EGCG-3-O-methyl, was shown to suppress postprandial hypertriglyceridemia in rats ${ }^{20)}$. Taken together, these findings indicate that these components might be associated with the strong lipase inhibitory activity of SR observed in the present study.

On the other hand, the EEXs did not produce marked lipase inhibition, despite containing high percentages of EGCG and ECG. According to Wang et al., the increasing of lipase inhibitory activity with catechins depended on the ratio of catechins to lipase and reach to the limit at the constant level $^{15)}$. Furthermore, lipase activity was also influenced by the emulsion particle size, which was determined by a number of factors such as mineral and emulsifier levels ${ }^{21)}$. Therefore, the EEX-mediated inhibition of lipase might be suppressed by the catechin:lipase ratio and the level of emulsification.

Some previous reports have indicated that anthocyaninrich foods can inhibit lipase activity ${ }^{22-24)}$. In this study, anthocyanin-rich SRs produced strong lipase inhibition, as compared with YK. However, even though SR2 contained high levels of anthocyanins, it did not show the strongest lipase inhibitory activity. According to You et al., the 50\% inhibitory concentrations of cyanidin and cyanidin 3,5 diglucoside against lipase were 0.17 and $0.89 \mathrm{mg} / \mathrm{mL}$, respectively ${ }^{22)}$. These concentrations were higher than the anthocyanin concentrations detected in the SR extracts studied in the present study. Therefore, the anthocyanin level showed a poor correlation with the lipase inhibitory effects of SR extracts. However, our in vivo study showed that SR2 puree powder intake suppressed the increases in plasma TG and NEFA concentrations observed after oral administration of oil. This result inferred that SR2 puree can inhibit lipase activity in vivo and suppress fat absorption from the small intestine ${ }^{25)}$. Gallate-type catechins such as EGCG may represent one of the lipase inhibitory components of SR.

\section{CONCLUSION}

SR tea may produce greater lipase inhibition than the general YK green tea variety. Simultaneous intake of fat 
and SR2 puree suppressed in vivo fat absorption in mice.

\section{Acknowledgements}

Part of this study was supported by funding from the Ministry of Agriculture, Forestry and Fisheries (Project 23005). We thank Atsushi Nesumi (NARO) for contributing to this study and Koji Yashima(Nepuree Corporation., Tokyo, Japan) for supplying the sample puree. We would like to thank Editage (www.editage.jp) for English language editing.

\section{References}

1) Kawser Hossain, M.; Abdal Dayem, A.; Han, J.; Yin, Y.; Kim, K.; Kumar Saha, S.; Yang, G.M.; Choi, H.Y.; Cho, S.G. Molecular mechanisms of the anti-obesity and anti-diabetic properties of flavonoids. Int. J. Mol. Sci. 17, 569 (2016).

2) Patel, S.A.; Ali, M.K.; Alam, D.; Yan, L.L.; Levitt, N.S.; Bernabe-Ortiz, A.; Checkley, W.; Wu, Y.; Irazola, V.; Gutierrez, L.; Rubinstein, A.; Shivashankar, R.; Li, X.; Miranda, J.J.; Chowdhury, M.A.; Siddiquee, A.T.; Gaziano, T.A.; Kadir, M.M.; Prabhakaran, D. Obesity and its relation with diabetes and hypertension: a crosssectional study across 4 geographical regions. Glob. Heart 11, 71-79 (2016).

3) Petzke, K.J.; Freudenberg, A.; Klaus, S. Beyond the role of dietary protein and amino acids in the prevention of diet-induced obesity. Int. J. Mol. Sci. 15, 13741391 (2014).

4) Tsuda, T. Recent progress in anti-obesity and anti-diabetes effect of berries. Antioxidants (Basel)5, E13 (2016).

5) Bender, N.; Portmann, M.; Heg, Z.; Hofmann, K.; Zwahlen, M.; Egger, M. Fish or n3-PUFA intake and body composition: a systematic review and meta-analysis. Obes. Rev. 15, 657-665(2014).

6) Higgins, J.A. Resistant starch and energy balance: impact on weight loss and maintenance. Crit. Rev. Food Sci. Nutr. 54, 1158-1166(2014).

7) Huang, J.; Wang, Y.; Xie, Z.; Zhou, Y.; Zhang, Y.; Wan, X. The anti-obesity effects of green tea in human intervention and basic molecular studies. Eur. J. Clin. Nutr. 68, 1075-1087(2014).

8) Nakaishi, H.; Matsumoto, H.; Tominaga, S.; Hirayama, M. Effects of black current anthocyanoside intake on dark adaptation and VDT work-induced transient refractive alteration in healthy humans. Altern. Med. Rev. 5, 553-562 (2000).

9) Kuntz, S.; Kunz, C.; Herrmann, J.; Borsch, C.H.; Abel, G.; Fröhling, B.; Dietrich, H.; Rudloff, S. Anthocyanins from fruit juices improve the antioxidant status of healthy young female volunteers without affecting anti-inflammatory parameters: results from the randomised, double-blind, placebo-controlled, cross-over ANTHONIA (ANTHOcyanins in Nutrition Investigation Alliance) study. Br. J. Nutr. 112, 925-936(2014).

10) Wang, Y.; Zhao, L.; Wang, D.; Huo, Y.; Ji, B. Anthocyanin-rich extracts from blackberry, wild blueberry, strawberry, and chokeberry: antioxidant activity and inhibitory effect on oleic acid-induced hepatic steatosis in vitro. J. Sci. Food Agric. 96, 2494-2503 (2016).

11) Tanaka, J.; Nakanishi, T.; Ogawa, K.; Tsuruma, K.; Shimazawa, M.; Shimoda, H.; Hara, H. Purple rice extract and anthocyanidins of the constituents protect against light-induced retinal damage in vitro and in vivo. J. Agric. Food Chem. 59, 528-536 (2011).

12) Maeda-Yamamoto, M.; Saito, T.; Nesumi, A.; Tokuda, Y.; Ema, K.; Honma, D.; Ogino, A.; Monobe, M.; Murakami, A.; Murakami, A.; Tachibana, H. Chemical analysis and acetylcholinesterase inhibitory effect of anthocyaninrich red leaf tea (cv. Sunrouge). J. Sci. Food Agric. 92, 2379-2386 (2012).

13) Oki, T.; Yusuke Sawai, Y.; Sato-Furukawa, M.; Suda, I. Validation of $\mathrm{pH}$ differential method for the determination of total anthocyanin content in black rice and black soybean with interlaboratory comparison. Bunseki Kagaku 60, 819-824 (2011).

14) Han, L.K.; Xu, B.J.; Kimura, Y.; Zheng, Y.; Okuda, H. Platycodi radix affects lipid metabolism in mice with high fat diet-induced obesity. J. Nutr. 130, 2760-2764 (2000).

15) Wang, S.; Sun, Z.; Dong, S.; Liu, Y.; Liu, Y. Molecular interactions between (-)-epigallocatechin gallate analogs and pancreatic lipase. PLoS One 9, e111143 (2014).

16) Maiti, T.K.; Ghosh, K.S.; Dasgupta, S. Interaction of(-) -epigallocatechin-3-gallate with human serum albumin: fluorescence, Fourier transform infrared, circular dichroism, and docking studies. Proteins 64, 355-362 (2006).

17) Wu, X.; He, W.; Yao, L.; Zhang, H.; Liu, Z.; Wang, W.; Ye, Y.; Cao, J. Characterization of binding interactions of (-) -epigallocatechin-3-gallate from green tea and lipase. J. Agric. Food Chem. 61, 8829-8835 (2013).

18) Gao, D.F.; Zhang, Y.J.; Yang, C.R.; Chen, K.K.; Jiang, H. J. Phenolic antioxidants from green tea produced from Camellia taliensis. J. Agric. Food Chem. 56, 75177521 (2008).

19) Huang, Y.L.; Tsujita, T.; Tanaka, T.; Matsuo, Y.; Kouno, I.; Li, D.P.; Nonaka, G. Triterpene hexahydroxydiphenoyl esters and a quinic acid purpurogallin carbonyl ester from the leaves of Castanopsis fissa. Phytochemistry 72, 2006-2014(2011).

20) Egashira, Y.; Kamohara, T.; Yamaguchi, W.; Irie, H.; 
Toyoda, Y.; Hanamura, M.; Hirai, S.; Shinoda, Y.; Maeda-Yamamoto, M. Suppression of postprandial hypertriglyceridemia in rats by Benifuuki tea extract. Nippon Shokuhin Kagaku Kogaku Kaishi 60, 407-411 (2013).

21) Shishikura, Y.; Khokhar, S.; Murray, B.S. Effects of tea polyphenols on emulsification of olive oil in a small intestine model system. J. Agric. Food Chem. 54, 19061913 (2006).

22) You, Q.; Chen, F.; Wang, X.; Luo, P.G.; Jiang, Y. Inhibitory effects of muscadine anthocyanins on $\alpha$-glucosidase and pancreatic lipase activities. J. Agric. Food Chem. 59, 9506-9511 (2011).

23) Sreerama, Y.N.; Takahashi, Y.; Yamaki, K. Phenolic an- tioxidants in some Vigna species of legumes and their distinct inhibitory effects on $\alpha$-glucosidase and pancreatic lipase activities. J. Food Sci. 77, C927-933 (2012).

24) Fabroni, S.; Ballistreri, G.; Amenta, M.; Romeo, F.V.; Rapisarda, P. Screening of the anthocyanin profile and in vitro pancreatic lipase inhibition by anthocyanincontaining extracts of fruits, vegetables, legumes and cereals. J. Sci. Food Agric. 96, 4713-4723 (2016).

25) Ogawa, A.; Kadooka, Y.; Kato, K.; Shirouchi, B.; Sato, M. Lactobacillus gasseri SBT2055 reduces postprandial and fasting serum non-esterified fatty acid levels in Japanese hypertriacylglycerolemic subjects. Lipids Health Dis. 13, 36 (2014). 